\title{
GMM-MUD: An Effective Multiuser Detection Algorithm for DS-UWB-Based Space Formation Flying Systems
}

\author{
Bo Ma, ${ }^{1,2}$ Mingyang Wu $\mathbb{D},{ }^{1}$ Zhilu Wu $\mathbb{D},{ }^{1}$ Zhendong Yin $\mathbb{D},{ }^{1}$ and Tao Shen ${ }^{1}$ \\ ${ }^{1}$ School of Electronics and Information Engineering, Harbin Institute of Technology, Harbin, China \\ ${ }^{2}$ China Spacesat Co. Ltd., Beijing, China \\ Correspondence should be addressed to Mingyang Wu; 16b905033@stu.hit.edu.cn
}

Received 31 May 2019; Revised 15 August 2019; Accepted 1 November 2019; Published 20 November 2019

Academic Editor: Krzysztof S. Kulpa

Copyright (c) 2019 Bo Ma et al. This is an open access article distributed under the Creative Commons Attribution License, which permits unrestricted use, distribution, and reproduction in any medium, provided the original work is properly cited.

In this paper, an effective multiuser detection (MUD) is proposed for direct sequence ultrawideband- (DS-UWB-) based space formation flying systems. The proposed method called GMM-MUD is based on Gaussian mixture models (GMMs) to suppress multiple access interference. The GMM describes probability distributions of the hypothesis testing problem which is used for bit classification. To reveal the difference between correct bits and error bits, the preprocessing operation applies a mapping function based on optimal multiuser detection. The parameters of GMM are estimated by using expectation-maximization (EM) algorithm. EM algorithm employs iterative operation to simplify the complexity of maximum likelihood estimation method and considers the mapping values of received bits as the observations. Simulation results demonstrate that the proposed GMM-MUD algorithm achieves good performances in terms of bit error rate performance, user capacity, and near-far resistance. Moreover, the computational complexity is low enough for space formation flying applications.

\section{Introduction}

In recent years, space formation flying has drawn a great deal of interests for missions in and beyond Lower Earth Orbit (LEO) [1]. Unlike a single large satellite, space formation flying is an effective and flexible mode of multiple small satellites, in which small satellites remain in a relatively stable distance and orientation and maintain connection using intersatellite communications. The common types of formation configurations include trailing, cluster, and constellation formation flying pattern. And the orbit of spacecraft can be considered as Keplerian orbits (KO) or non-Keplerian orbits (NKO) [2-4]. The different formation configurations and orbit models need different parameters such as the frequency allocation, data rate, modulation schemes, power control, and antenna design, which affect the performance of the intersatellite communications. The efficient and reliable intersatellite communications enable time synchronization and autonomous control of the attitude and position. However, the communication links are influenced by the limitations of small satellites such as limited power, antenna size, computing capabilities, and intermittent channels [5]. Due to the advantages of impulseradio ultrawideband (IR-UWB) technology, UWB is a suitable choice for the physical layer of intersatellite communications $[6,7]$.

In some space formation flying applications, the number of small satellites is often very large. For example, the QB-50 mission funded by the European Union has 50 small satellites flying in formation and the MAGNAS system comprises 4 space formation swarms, with 6 small satellites and 1 mother spacecraft in each swarm [5]. In order to ensure the reliability and validity of multisatellite communications, the multiple access interference (MAI) suppression is the key technique that we consider in this paper.

Multiple access approaches are necessary MAI suppression techniques at transmitters. Time hopping multiple access (THMA) and direct sequence multiple access (DSMA) are considered as the common multiple access approaches for IR-UWB systems. In the time hopping ultrawideband (TH-UWB) system, each symbol is represented by a sequence of pulses with specific relative delays 
which are determined by the $\mathrm{TH}$ code assigned to each user. Unlike THMA scheme, DSMA is similar to code division multiple access (CDMA) which improves multiple access potential and user capacity by applying orthogonal pseudo noise (PN) codes [8]. MA capabilities of both schemes are provided by using different sequences for different users. In this paper, we consider the DS-UWB as the physical layer technology for space formation flying. However, MAI cannot be completely suppressed by using DSMA scheme owing to the incomplete orthogonality of PN codes and large user number. Concerning this problem, multiuser detection (MUD) is applied as an effective MAI suppression method at receivers. Consequently, the MUD algorithm is the key technique which is essential to cope with MAI for DS-UWB systems [9].

In this paper, we propose a Gaussian mixture model(GMM-) based multiuser detection algorithm which is called GMM-MUD for DS-UWB-based space formation flying systems. We first establish the system model of space formation flying based on DS-UWB, and the outer space channel is considered as the additive white Gaussian noise (AWGN) channel. Concerning the DS-UWB system model, multiuser signals are received by using a set of matched filters corresponding to each user which utilize the correlations between received signals and the template signals. The received bits may be error due to MAI and channel noise. A preprocessing method which is called bit mapping (BM) is applied to the multiuser detector to map the received bits to the mapping space. The probability distributions of error bits and correct bits in mapping space are different obviously. We use GMM to represent the different distributions of error bits and correct bits in mapping space. In order to estimate parameters in GMM, expectation-maximization (EM) iterative algorithm is used in this paper. Then, the threshold value of received bits considered as error bits can be given based on the GMM. Hence, we distinguish and correct the error bits to improve the performances in terms of bit error rate (BER), user capacity, and near-far resistance which are validated by computer simulations.

The rest of this paper is organized as follows. In Section 2, we provide a review of existing works on MUD algorithms. In Section 3, we describe the model of DS-UWB system, including the transmitter model, the intersatellite communication links, and the receiver model. In Section 4, the GMM-MUD algorithm is proposed and the block diagram of GMM-based multiuser detector is given. The simulation results and discussion are shown in Section 5. Finally, Section 6 concludes this paper.

\section{Related Work}

There has been a great quantity of literature on MUD to suppress MAI at receivers. A conventional multiuser detector consists of a set of matched filters corresponding to different users followed by multiuser detectors based on various MUD algorithms. The matched filter can be considered as the simplest multiuser detector termed as single-user detection (SUD) with the multiple access scheme applied to communication systems [10]. In this case, the desired signal can be detected by using the correlation between the desired signal and the corresponding template signal, and the interfering signals are considered as noise. Unlike the single-user detector, the multiuser detector jointly detects all signals transmitted from multiple users by using MUD algorithm. Verdú proposed optimal multiuser detection (OMD) based on the maximum likelihood sequence estimator (MLSE) to minimize the probability of error bits and achieve the best BER performance [11]. However, the complexity of OMD is exponential in the number of users. To cope with the complexity problem, numerous suboptimal MUD algorithms with the balance between performance and complexity have been proposed, including linear detectors and nonlinear detectors.

Linear multiuser detectors use a linear transform matrix to transform the output of matched filter. The decorrelating detector which is also known as zero-forcing (ZF) detector [12] can completely suppress MAI in the absence of noise. However, the ZF detector may amplify noise when the correlation matrix is not equal to identity matrix. The minimum mean square error (MMSE) detector [13] takes into account the influence of both MAI and noise and tries to maximize the signal to interference plus noise ratio (SINR). In the case of low and medium SNR, MMSE detector reduces the influence of noise amplification. In [14], a MMSE detector with low computational complexity was proposed for TH-UWB systems, in which the detector only considers the users whose pulses collide with the desired user. To cope with the problem of dynamic multiple access channel, adaptive MUD algorithm was proposed by using some optimization algorithms, such as gradient descent algorithm and recursive least squares (RLS) [15]. A blind adaptive linear detector $[16,17]$ was proposed without any training sequences to achieve higher spectrum efficiency.

Successive interference cancellation (SIC) detector is the nonlinear MUD detector with the iterative method [18]. The idea of SIC is that signals of multiple users are successively detected. Another subtractive MAI cancellation method is parallel interference cancellation (PIC) [19], in which the users' signals are detected in parallel and further estimated after subtracting all the MAI estimated signals from the received signal. Neural networks-based MUD algorithms have been proposed in $[20,21]$. Moreover, biological intelligence methods, such as genetic algorithms, ant colony optimization, and artificial fish swarm algorithm, have been studied to solve the optimization problem in [22-24].

In [25], MUD problem is formulated as a maximum a posteriori (MAP) estimation problem. Due to the NP-hard problem of MAP solution, the nonconvex optimization problem has been transformed into convex relaxation optimization problem which can be solved by using optimization tools with low complexity. In recent years, the MUD algorithms based on compressed sensing (CS) have been proposed $[26,27]$. When the number of active users is much smaller than the total user number in communication systems, the reconstruction of the transmitted signals based on CS algorithm can reduce the number of correlators and the computational complexity. In [28], the authors have proposed two MUD detectors based on CS, including the reduced-dimension decorrelating (RDD) detector and reduced-dimension decision-feedback (RDDF) detector. 
These studies propose various MUD algorithms with good performances and their own advantages as well as disadvantages. In a word, the tradeoff problem between computational complexity and MUD performance is always the research objective.

\section{System Model}

We consider a $K$-user space formation flying system based on DS-UWB, where each transmitter employs binary phase shift keying (BPSK) modulation. The transmitted signal of the $k$ th user (where $k=1,2, \ldots, K$ ) can be written as

$$
x_{k}(t)=\sqrt{E_{k}} \sum_{i=0}^{P-1} \sum_{j=0}^{N_{c}-1} b_{k}^{i} c_{k}^{j} p\left(t-i T_{\mathrm{s}}-j T_{\mathrm{c}}\right),
$$

where $E_{k}$ is the transmission energy of the $k$ th user and $b_{k}^{i} \in\{-1,1\}$ is the $i$ th BPSK modulated symbol which is spread with the corresponding PN code $c_{k} \in\{-1,1\}$. $p(t)$ denotes the transmitted pulse carrying the $k$ th user's information, which often adopts the second derivative of a Gaussian pulse with a pulse duration of a nanosecond. $P$ is the number of bits per packet, and $N_{c}$ denotes the length of the $\mathrm{PN}$ sequence. Moreover, $T_{s}$ and $T_{c}$ are symbol duration and chip duration, respectively. Note that $N_{c}$ equals to $T_{s} / T_{c}$.

In normal conditions, the UWB channel model suffers effects of dense multipath due to the large bandwidth of the transmitted signal. The channel impulse response can be written as

$$
h(t)=\sum_{l=1}^{L} \alpha_{l} \delta\left(t-\tau_{l}\right)
$$

where $L$ denotes the number of multipath components. $\alpha_{l}$ and $\tau_{l}$ are the amplitude coefficient and channel delay of the lth multipath component, respectively. The statistical parameters of the spacecraft channel model depend on orbital constraints, time delay, and path loss. In this paper, we consider the outer space as the communication scenario. For simplicity, the free space channel can be approximately considered as the additive white Gaussian noise (AWGN) channel without regard to multipath. The dense multipath channel model applied to the indoor short-range UWB communications is not concerned. Hence, the parameters of the channel model depend on the distance between spacecraft and equipment time delay and the number of multipath components is assumed to be 1 . The received signal through AWGN channels can be expressed as

$$
r(t)=\sum_{k=1}^{K} \sum_{i=0}^{P-1} \sum_{j=0}^{N_{c}-1} \alpha_{k} \sqrt{E_{k}} b_{k}^{i} c_{k}^{j} p\left(t-i T_{\mathrm{s}}-j T_{\mathrm{c}}-\tau_{k}\right)+n(t),
$$

where $a_{k}$ and $\tau_{k}$ denote the channel fading and the time delay of the $k$ th user, respectively. And $n(t)$ is zero-mean AWGN with two-sided power spectral density $N_{0} / 2 \mathrm{~W} / \mathrm{Hz}$.

The receiver consists of a set of matched filters corresponding to each user. The received signal $r(t)$ is the input of the group of matched filters. The template signal $m_{l}(t)$ of the $l$ th matched filter is used for coherent reception, which can be written as

$$
m_{l}(t)=\sum_{n=0}^{N_{\mathrm{c}}-1} c_{l}^{n} p\left(t-n T_{\mathrm{c}}\right)
$$

For simplicity, we consider the received signal in one symbol duration and assume that $\alpha_{k} \sqrt{E_{k}}=A_{k}$. The output of the $l$ th- (where $l=1,2, \ldots, K$ ) matched filter is expressed as

$$
\begin{aligned}
y_{l}= & \int_{T_{\mathrm{s}}} r(t) m\left(t-\tau_{l}\right) d t \\
= & \int_{T_{\mathrm{s}}}\left(\sum_{k=1}^{K} \sum_{j=0}^{N_{\mathrm{c}}-1} A_{k} b_{k}^{i} c_{k}^{j} p\left(t-j T_{\mathrm{c}}-\tau_{k}\right)+n(t)\right) \\
& \times \sum_{n=0}^{N_{\mathrm{c}}-1} c_{l}^{n} p\left(t-n T_{\mathrm{c}}-\tau_{l}\right) d t \\
= & A_{l} b_{l} r_{l l}+\sum_{k=1, k \neq l}^{K} A_{k} b_{k} r_{k l}+n_{l} .
\end{aligned}
$$

The first term of (5) denotes the coherent reception of the desired user, the second term is the MAI caused by incomplete orthogonality of PN codes, and the last term is the noise interference. Furthermore, the correlation value $r_{k l}$ between user $k$ and user $l$ is given by

$$
r_{k l}=\int_{T_{\mathrm{s}}} \sum_{j=0}^{N_{\mathrm{c}}-1} c_{k}^{j} p\left(t-j T_{\mathrm{c}}-\tau_{k}\right) \times \sum_{n=0}^{N_{\mathrm{c}}-1} c_{l}^{n} p\left(t-n T_{\mathrm{c}}-\tau_{l}\right) d t .
$$

The noise inference term $n_{l}$ is expressed as

$$
\begin{aligned}
n_{l} & =\int_{T_{\mathrm{s}}} n(t) \times \sum_{n=0}^{N_{\mathrm{c}}-1} c_{l}^{n} p\left(t-n T_{\mathrm{c}}-\tau_{l}\right) d t \\
& =\sum_{n=0}^{N_{\mathrm{c}}-1} c_{l}^{n} \int_{T_{\mathrm{s}}} n(t) \times p\left(t-n T_{\mathrm{c}}\right) d t .
\end{aligned}
$$

Obviously, samples of $n_{l}$ obey a Gaussian distribution, whose mean value and variance are calculated as follows:

$$
\begin{aligned}
E\left[n_{l}\right]= & \sum_{n=0}^{N_{\mathrm{c}}-1} c_{l}^{n} \int_{T_{\mathrm{s}}} E[n(t)] \times p\left(t-n T_{\mathrm{c}}\right) d t=0 \\
D\left[n_{l}\right]= & E\left[n_{l}\right]^{2}-E^{2}\left[n_{l}\right]=E\left[n_{l}\right]^{2} \\
= & E\left[\sum_{m=0}^{N_{\mathrm{s}}-1} \sum_{n=0}^{N_{\mathrm{s}}-1} c_{l}^{m} c_{l}^{n} \int_{-\infty}^{\infty} \int_{-\infty}^{\infty} n\left(t_{1}-m T_{\mathrm{c}}\right)\right. \\
& \left.\times p\left(t_{1}\right) \times n\left(t_{2}-n T_{\mathrm{c}}\right) \times p\left(t_{2}\right) d t_{1} d t_{2}\right] \\
= & \frac{N_{0}}{2} \sum_{m=0}^{N_{\mathrm{s}}-1} \sum_{n=0}^{N_{\mathrm{s}}-1} c_{l}^{m} c_{l}^{n} \int_{-\infty}^{\infty} p(t) \times p\left(t+(m-n) T_{\mathrm{c}}\right) d t \\
= & \frac{N_{0} N_{\mathrm{c}} E_{l}}{2} .
\end{aligned}
$$


Consequently, the $K$-dimensional y vector can be rewritten as

$$
\mathbf{y}=\mathbf{R A b}+\mathbf{n},
$$

where matrix $\mathbf{R}=\left(r_{k l}\right)_{K \times K}$ denotes the cross-correlation matrix, matrix $\mathbf{A}=\operatorname{diag}\left(A_{1}, A_{2}, \ldots, A_{K}\right)$ denotes the amplitude matrix, vector $\mathbf{b}=\left[b_{1}, b_{2}, \ldots, b_{K}\right]^{\mathrm{T}}$ denotes the transmitted bits, and vector $\mathbf{n}=\left[n_{1}, n_{2}, \ldots, n_{K}\right]^{\mathrm{T}}$ denotes the noise interference.

After these matched filters, the output $y$ should be judged by the sign detector as follows:

$$
\widehat{\mathbf{b}}=\operatorname{sgn}(\mathbf{y}) .
$$

Figure 1 shows the frameworks of the DS-UWB system. The multiuser detector signed by using a dashed box is applied after the correlation receiver to further suppress MAI. Note that the MUD algorithm is mainly studied in this paper.

\section{GMM-MUD Algorithm}

4.1. Signal Preprocessing. The output of the $l$ th matched filter $y_{l}$ in (5) consists of three terms, which is hard to estimate the probability distribution owing to the uncertain transmitted bits and correlation values. Hence, we preprocess the detected bits judged by the sign detector in order to reveal the influence of MAI and study the statistical property of the received signals. The signal preprocessing method is called bit mapping, which employs a mapping function to map the received bits into the mapping space [29]. The mapping function is based on the theory of OMD. The optimal solution to OMD is given by

$$
\widehat{\mathbf{b}}_{O M D}=\arg \left\{\max _{\widehat{\mathbf{b}} \in\{-1,+1\}}\left(2 \widehat{\mathbf{b}}^{\mathrm{T}} \mathbf{A} \mathbf{y}-\widehat{\mathbf{b}}^{\mathrm{T}} \mathbf{A R A} \widehat{\mathbf{b}}\right)\right\} .
$$
that

In order to solve the optimization problem, we assume

$$
F(\widehat{\mathbf{b}})=\frac{1}{2} \widehat{\mathbf{b}}^{\mathrm{T}} \mathbf{A} \mathbf{R} \mathbf{A} \widehat{\mathbf{b}}-\widehat{\mathbf{b}}^{\mathrm{T}} \mathbf{A y}
$$

According to (11), the optimization problem of OMD is equal to the solution to $\mathbf{b}$ that minimizes the function $F(\widehat{\mathbf{b}})$. We can rewrite (12) as

$$
F(\widehat{\mathbf{b}})=\frac{1}{2} \sum_{i=1}^{K} \sum_{j=1}^{K} A_{i} A_{j} r_{i j} \widehat{\mathbf{b}}_{i} \widehat{\mathbf{b}}_{j}-\sum_{i=1}^{K} \widehat{\mathbf{b}}_{i} A_{i} y_{i} .
$$

In order to reduce the complexity of $F(\widehat{\mathbf{b}})$ and construct a mapping function, we calculate the derivative of $F(\widehat{\mathbf{b}})$ as follows:

$$
\frac{\partial F}{\partial \widehat{\mathbf{b}}}=\mathbf{A R A} \widehat{\mathbf{b}}-\mathbf{A y}
$$

To expand (14), we get $K$-order linear equations written as follows:

$$
\left\{\begin{array}{c}
\frac{\partial F}{\partial \hat{b}_{1}}=\sum_{k=1}^{K} A_{1} A_{k} r_{1 k} \widehat{b}_{k}-A_{1} y_{1}, \\
\frac{\partial F}{\partial \widehat{b}_{2}}=\sum_{k=1}^{K} A_{2} A_{k} r_{2 k} \widehat{b}_{k}-A_{2} y_{2}, \\
\vdots \\
\frac{\partial F}{\partial \widehat{b}_{K}}=\sum_{k=1}^{K} A_{K} A_{k} r_{K k} \widehat{b}_{K}-A_{K} y_{K} .
\end{array}\right.
$$

The mapping function based on (15) is assumed as

$$
\Phi\left(\widehat{b}_{j}\right)=\frac{\partial F}{\partial \widehat{b}_{j}} \widehat{b}_{j}=\widehat{b}_{j}\left(\sum_{k=1}^{K} A_{j} A_{k} r_{j k} \widehat{b}_{k}-A_{j} y_{j}\right) \text {. }
$$

The mapping function $\Phi\left(\widehat{b}_{j}\right)$ denotes the first partial derivative of $F(\widehat{b})$ with respect to $\widehat{b}_{j}$ multiplied by $\widehat{b}_{j}$. Substituting (5) into (16), we can get

$$
\begin{aligned}
\Phi\left(\widehat{b}_{j}\right)= & A_{j} \widehat{b}_{j}\left[A_{j} r_{j j}\left(\widehat{b}_{j}-b_{j}\right)+\sum_{k=1, k \neq j}^{K} A_{k} r_{k j}\left(\widehat{b}_{k}-b_{k}\right)-n_{j}\right] \\
= & A_{j} A_{j} r_{j j}\left(\widehat{b}_{j}-b_{j}\right) \widehat{b}_{j}+\sum_{k=1, k \neq j}^{K} A_{j} A_{k} r_{k j}\left(\widehat{b}_{k}-b_{k}\right) \widehat{b}_{j} \\
& -A_{j} n_{j} \widehat{b}_{j} .
\end{aligned}
$$

The mapping function reflects the difference between the mapping values of error bits and correct bits. Every item on the right side of the equation has $A_{j}$. Hence, $A_{j}$ cannot affect the difference between the mapping values of error bits and correct bits. Both sides are divided by $A_{j}$ and (17) can be rewritten as

$$
\bar{\Phi}\left(\widehat{b}_{j}\right)=A_{j} r_{j j}\left(\widehat{b}_{j}-b_{j}\right) \widehat{b}_{j}+\sum_{k=1, k \neq j}^{K} A_{k} r_{k j}\left(\widehat{b}_{k}-b_{k}\right) \widehat{b}_{j}+n_{j} \widehat{b}_{j} \text {. }
$$

In order to judge whether the detected bit $\widehat{b}_{j}$ is correct or not, we can analyze the problem by using hypothesis testing. We state the null and alternative hypotheses as follows:

$$
\begin{cases}H_{0}, & \widehat{b}_{j} \text { is the correct bit, } \\ H_{1}, & \widehat{b}_{j} \text { is the error bit. }\end{cases}
$$

If hypothesis $H_{0}$ is accepted, we can get $\widehat{b}_{j}=b_{j}$. If hypothesis $H_{1}$ is accepted, $\widehat{b}_{j}=-b_{j}$. Hence, probability of the mapping value in hypothesis testing can be expressed as

$$
\left\{\begin{array}{l}
\Phi\left(\widehat{b}_{j}\right) \mid H_{0} \sim N\left(\sum_{k=1, k \neq j}^{K} A_{k} r_{k j}\left(\widehat{b}_{k}-b_{k}\right) \widehat{b}_{j}, \frac{N_{0} N_{c} E_{l}}{2}\right), \\
\Phi\left(\widehat{b}_{j}\right) \mid H_{1} \sim N\left(2 A_{j} r_{j j}+\sum_{k=1, k \neq j}^{K} A_{k} r_{k j}\left(\widehat{b}_{k}-b_{k}\right) \widehat{b}_{j}, \frac{N_{0} N_{c} E_{l}}{2}\right) .
\end{array}\right.
$$




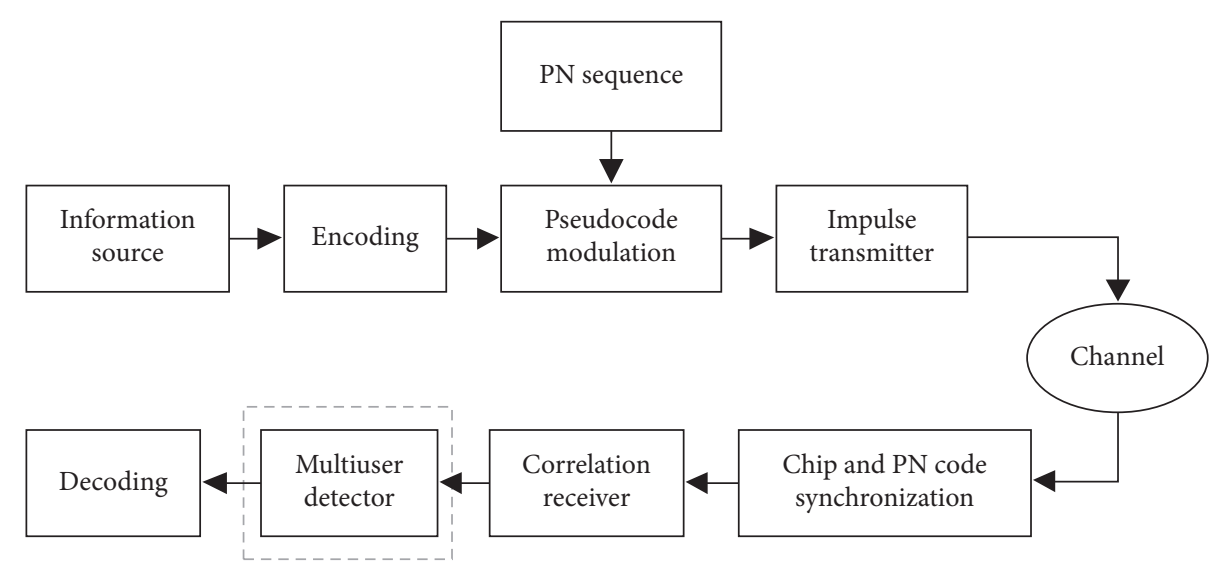

FIGURE 1: Framework of the DS-UWB system. The frameworks of the DS-UWB system include the transmitter model, the intersatellite communication links, and the receiver model.

In common situations, there are few error bits detected by using matched filters. We can deduce that $r_{j j} \approx 1$ and $r_{j j} \gg r_{k j}$ owing to DS codes for different users. The schematic diagram of probability density distribution of mapping values in hypothesis testing is shown in Figure 2.

It is obvious that the mean value of the normal distribution approximates to 0 under the null hypothesis $H_{0}$. Under the alternative hypothesis $H_{1}$, the mean value is approximately equal to 2 . In order to analyze the probability distribution of mapping values and judge the error bits, we construct the Gaussian mixture model approximating mapping value distribution. Then, the EM algorithm is employed for simplifying difficult maximum likelihood problems and estimating the parameters of GMM. The proposed GMM-MUD algorithm is described in the following section.

4.2. GMM-Based MUD Algorithm. We use the Gaussian mixture model to approximate the distribution of mapping values to judge whether the detected bits are correct or not. GMM is suitable for the distribution of mapping values which can be described by a multimodal distribution. A GMM can be written as

$$
g(x)=\sum_{i=1}^{M} w_{i} p\left(x \mid \mu_{i}, \sigma_{i}^{2}\right)
$$

where $M$ is the number of Gaussian models, $w_{i}$ is the weight of the $i$ th Gaussian model, $\mu_{i}$ and $\sigma_{i}^{2}$ denote mean value and variance, respectively, and $x$ denotes the mapping value, i.e., $x=\bar{\Phi}\left(\widehat{b}_{j}\right)$. Hence, a Gaussian mixture model is a linear combination of $M$ Gaussian models weighted according to $\left\{w_{i}\right\}_{i=1}^{M}$. The parameter $\theta$ which is to be estimated is given by

$$
\theta=\left(w_{1}, \ldots, w_{M}, \mu_{1}, \ldots, \mu_{M}, \sigma_{1}^{2}, \ldots, \sigma_{M}^{2}\right) .
$$

Moreover, $\left\{w_{i}\right\}_{i=1}^{M}$ are imposed to be

$$
\begin{aligned}
w_{1}, \ldots, w_{M} & \geq 0, \\
\sum_{i=1}^{M} w_{i} & =1 .
\end{aligned}
$$

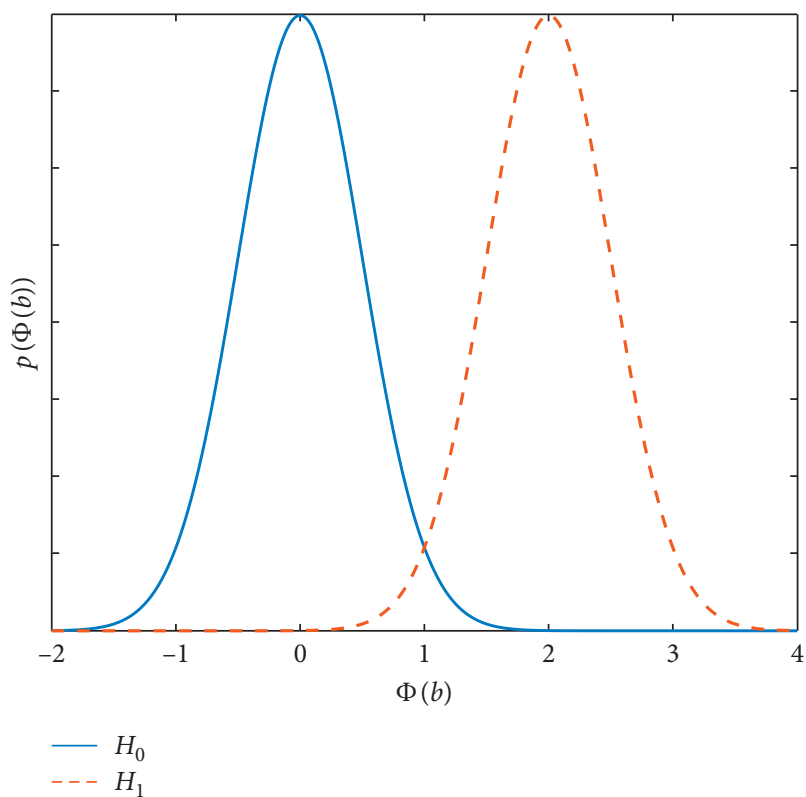

FIGURE 2: Schematic diagram of probability density distribution of $\Phi\left(\widehat{\mathbf{b}}_{j}\right)$. The curves represent the probability density distributions of mapping values in hypothesis testing.

The parameter $\theta$ in the Gaussian mixture model is estimated by maximum likelihood estimation (MLE). The likelihood can be expressed by

$$
L(\theta)=\prod_{t=1}^{N} g\left(x_{t} ; \theta\right) .
$$

MLE is used to maximize $L(\theta)$ with respect to $\theta$. MLE can be formulated as

$$
\begin{array}{ll}
\widehat{\theta}=\underset{\theta}{\arg \max } L(\theta) \\
\text { subject to } \quad w_{1}, \ldots, w_{M} \geq 0 \\
\sum_{i=1}^{M} w_{i}=1 .
\end{array}
$$


However, it is difficult to solve the MLE problem in practice. We solve this problem by means of EM algorithm which is an iterative algorithm.

The EM algorithm was originally developed for obtaining a maximum likelihood solution when input is partially observable. The log likelihood for the mapping values can be defined as

$$
\begin{aligned}
\log P(x, \gamma \mid \theta) & =\log \prod_{i=1}^{M} \prod_{t=1}^{N}\left[w_{i} p\left(x_{t} \mid \theta_{i}\right)\right]^{\gamma_{i t}} \\
& =\sum_{i=1}^{M} \sum_{t=1}^{N} \gamma_{i t} \log w_{i}+\sum_{i=1}^{M} \sum_{t=1}^{N} \gamma_{i t} \log p\left(x_{t} \mid \theta_{i}\right),
\end{aligned}
$$

where $n$ is the number of observed mapping values and $\gamma$ is an implicit parameter. Thus, $\gamma_{i t}$ can be written by

$$
\gamma_{m t}= \begin{cases}1, & t \text { th observation belonging to } m \text { th model, } \\ 0, & \text { otherwise }\end{cases}
$$

We define $\theta^{j}$ as the set of parameters for the $j$ th iteration operation of EM algorithm. EM algorithm is an iterative algorithm to estimate the ML with finite GMM parameters. At E-step of the EM algorithm, the $Q$ function is defined as

$$
\begin{aligned}
Q\left(\theta, \theta^{j}\right) & =E\left[\log P(x, \gamma \mid \theta) \mid y, \theta^{j}\right] \\
& =\sum_{i=1}^{M} \sum_{t=1}^{N} E\left(\gamma_{i t}\right) \log w_{i}+\sum_{i=1}^{M} \sum_{t=1}^{N} E\left(\gamma_{i t}\right) \log p\left(x_{t} \mid \theta_{i}\right),
\end{aligned}
$$

where $E\left(\gamma_{m t} \mid x, \theta\right)$ denotes the $m$ th model's responsibility for $t$ th observation. The responsibility is expressed as

$$
\begin{aligned}
\widehat{\gamma}_{m t} & =E\left(\gamma_{m t} \mid x_{t}, \theta\right)=P\left(\gamma_{m t}=1 \mid x_{t}, \theta\right) \\
& =\frac{P\left(\gamma_{m t}=1 \mid \theta\right) P\left(x_{t} \mid \gamma_{m t}=1, \theta\right)}{\sum_{i=1}^{M} P\left(\gamma_{i t}=1 \mid \theta\right) P\left(x_{t} \mid \gamma_{i t}=1, \theta\right)} \\
& =\frac{w_{m} P\left(x_{t} \mid \theta_{m}\right)}{\sum_{i=1}^{M} w_{i} p\left(x_{t} \mid \theta_{i}\right)} .
\end{aligned}
$$

At the M-step of EM algorithm, the parameter vector $\theta$ is estimated to ensure the maximum of the $Q$ function. The parameter $\theta$ is estimated by maximizing

$$
\theta^{j+1}=\arg \max _{\theta} Q\left(\theta, \theta^{j}\right)
$$

We calculate the partial derivative with respect to $\theta$ and set the partial derivative to zero. According to (28)-(30), the GMM parameters can obtained by

$$
\begin{aligned}
\widehat{\mu}_{m} & =\frac{\sum_{t=1}^{N} \widehat{\gamma}_{m t} x_{t}}{\sum_{t=1}^{N} \widehat{\gamma}_{m t}}, \\
\widehat{\sigma}_{m}^{2} & =\frac{\sum_{t=1}^{N} \widehat{\gamma}_{m t}\left(x_{t}-\widehat{\mu}_{m}\right)^{2}}{\sum_{t=1}^{N} \widehat{\gamma}_{m t}},
\end{aligned}
$$

$$
\widehat{w}_{m}=\frac{\sum_{t=1}^{N} \widehat{\gamma}_{m t}}{N}
$$

In this paper, the number of submodels is equal to 2 due to the hypothesis testing. After analyzing the EM algorithm, we provide a realization of the proposed GMM-MUD algorithm shown in Algorithm 1. The mapping values obtained by preprocessing are considered as the observations for EM algorithm. The parameters are estimated through iteration operation. We employ the GMM method to classify the mapping values of received bits into two submodels to judge whether the bits are correct or not. If the received bit is error, which means alternative hypothesis $\mathrm{H}_{1}$ is accepted, we revise the error bit by inverting the sign of bit. A block diagram of the proposed GMM-MUD detector is shown in Figure 3. Data preprocessing is based on mapping function in order to employ hypothesis testing with different probability distributions. GMM classification based on GMM is obtained by using multiuser signals and EM algorithm.

Moreover, the computational complexity of the proposed GMM-MUD algorithm mainly depends on the estimation of GMM parameters, which is much lower than that of OMD. It is obvious that the GMM-MUD algorithm is a linear relationship with the user number. Hence, the GMMMUD is suitable for suppressing MAI in practice.

\section{Results and Discussion}

In this section, simulation results are shown in order to validate the performances of the proposed GMM-MUD algorithm in terms of BER performance, user capacity, and near-far resistance under the assumption of perfect channel estimation.

The GMM-MUD algorithm is employed to an asynchronous multiuser BPSK DS-UWB system for space formation flying. We adopt the Kasami spreading codes with the length of 255 for different users to achieve MA capacity. The chip duration of per pulse $T_{c}$ is chosen to be $1 \mathrm{~ns}$. The number of submodel is assumed to be 2 . The free space channels between spacecraft are considered as the AWGN channels.

5.1. BER Performance. We assume that the space formation flying system is a collaborative communication system, in which each spacecraft obtains the information about the exact distance between each craft. After applying the power control technology, the farther spacecraft transmits signals with more transmitted power. The formation flying system ensures the signals from different users have equal received power in order to prevent the near-far effect. We evaluate the BER performance of the proposed GMM-MUD algorithm. The traditional MUD detector including matched filter (MF), decorrelating (DEC) detector, minimum mean square error (MMSE) detector, and optimal multiuser detector (OMD) is used for comparisons. We assume that there are 10 users in the DS-UWB system and SNR varies from 0 to $8 \mathrm{~dB}$. Here, we define SNR as the ratio of average energy per bit $E_{b}$ over the power spectrum density of AWGN $N_{0}$. Figure 4 shows the BER performance of the proposed algorithm. 
(1) Receive the multiuser signals by using matched filters and received bits are detected by using (9) and (10)

(2) Map the detected bits for preprocessing by using (18)

(3) Set the initial value of $\theta=\left(w_{1}, \ldots, w_{M}, \mu_{1}, \ldots, \mu_{M}, \sigma_{1}^{2}, \ldots, \sigma_{M}^{2}\right)$

(4) Expectation step: compute the responsibilities by using (29)

(5) Maximization step: compute the weights, mean values, and variances by using (31), (32), and (33)

(6) Iterate steps 4 and 5 until convergence

(7) Classify the received bits into two submodels after estimating the GMM parameters

(8) If hypothesis $H_{1}$ is accepted (the received bit is error), the error bit is revised by inverting the sign

Algorithm 1: GMM-MUD algorithm.

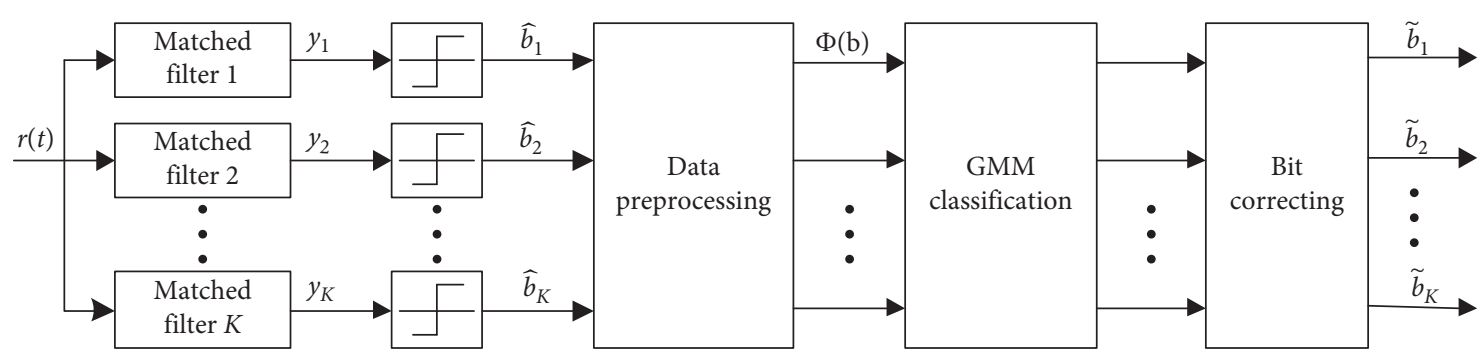

FIGURE 3: Block diagram of the GMM-MUD detector. GMM classification module based on GMM is implemented by using multiuser signals and EM algorithm.

The curve marked OMD is the theoretical lower limit of BER performance with the exponential complexity. The MF detector is able to utilize the MA scheme to achieve limited improvement of MA performance. Hence, the performance of MF is the worst among all the MUD detectors.

The DEC and MMSE detectors have almost the same BER performance which is much better than that of the MF detector. The proposed GMM-MUD algorithm outperforms the traditional suboptimal MUD detectors. And the GMM detector has a superior BER performance close to the OMD detector with 10 users. Note that the GMM-MUD detector has the linear computational complexity with respect to user number, which is much lower than OMD.

5.2. User Capacity. We evaluate the user capacity of DSUWB based on GMM-MUD algorithm by increasing the user number up to 20 and 30 . The user capacity reflects the number of available spacecraft communicating simultaneously in the same channel in the condition of specific SNR and BER.

Figure 5 indicates the BER performance of various detectors for DS-UWB with 20 users. The performances of suboptimal MUD detectors degenerate due to the increase of user number. More users in the DS-UWB system cause larger MAI. The MF detector can hardly suppress MAI due to the limited MA capacity of DS codes. However, the proposed GMM-MUD algorithm still has better BER performance approximating to OMD than DEC and MMSE detectors.

Figure 6 shows the BER performances with 30 users. Compared with Figures 4 and 5, the detectors exhibit gradual performance degradation as the number of users increase. Moreover, the GMM-MUD proposed in this paper has about $0.5 \mathrm{~dB}$ performance gain in comparison with the

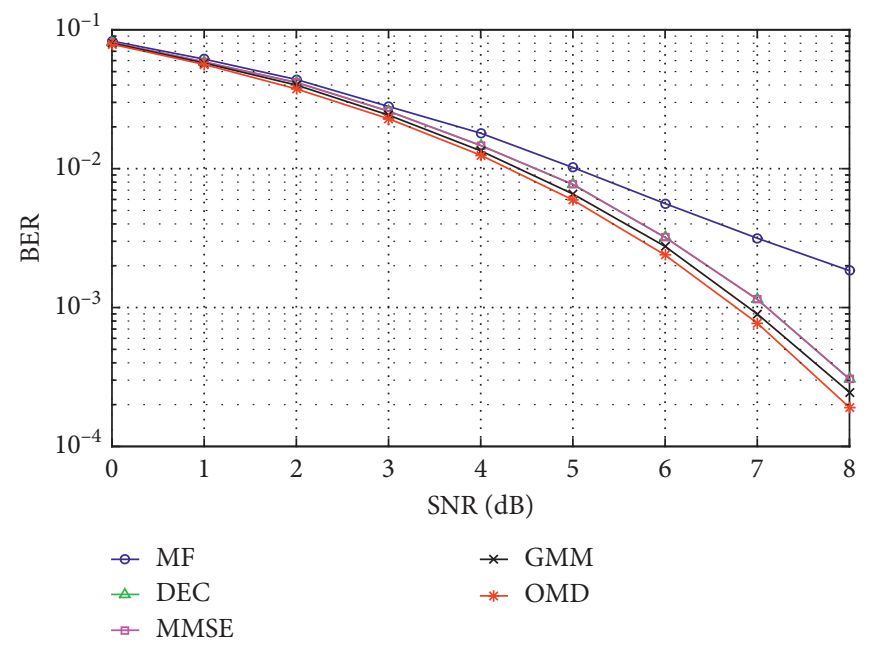

FIGURE 4: BER performance of various detectors with user number $K=10$. The curves represent the BER performance in the condition of different SNRs.

MMSE detector. According to Figures 4-6, we conclude that MMSE and DEC detectors are more sensitive to user number than the proposed GMM-MUD receiver. And the GMM-MUD algorithm becomes deteriorated due to the increase of user number. However, the performance of GMM-MUD deteriorates slightly and it is the best detector among all the traditional suboptimal MUD detectors.

In Figure 7, we evaluate the user capacity of DS-UWB system applying the GMM-MUD algorithm in the condition of $8 \mathrm{~dB}$ SNR. The BER performance of GMM-MUD is close to OMD in comparison with other algorithms. It demonstrates that the proposed algorithm can provide large user 


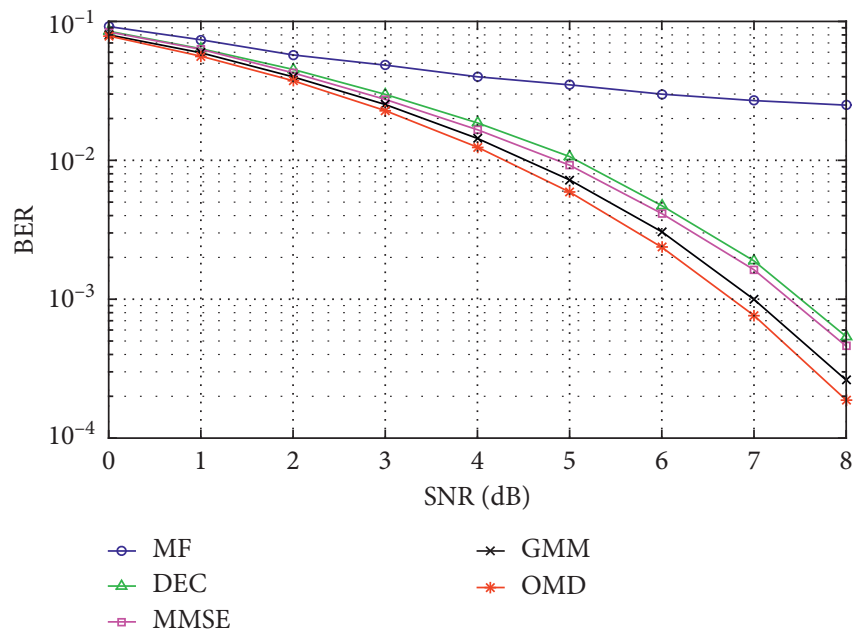

FIGURE 5: BER performance of various detectors with user number $K=20$. We compare Figure 5 (user number is 20) with Figure 6 (user number is 30 ) and Figure 4 (user number is 10).

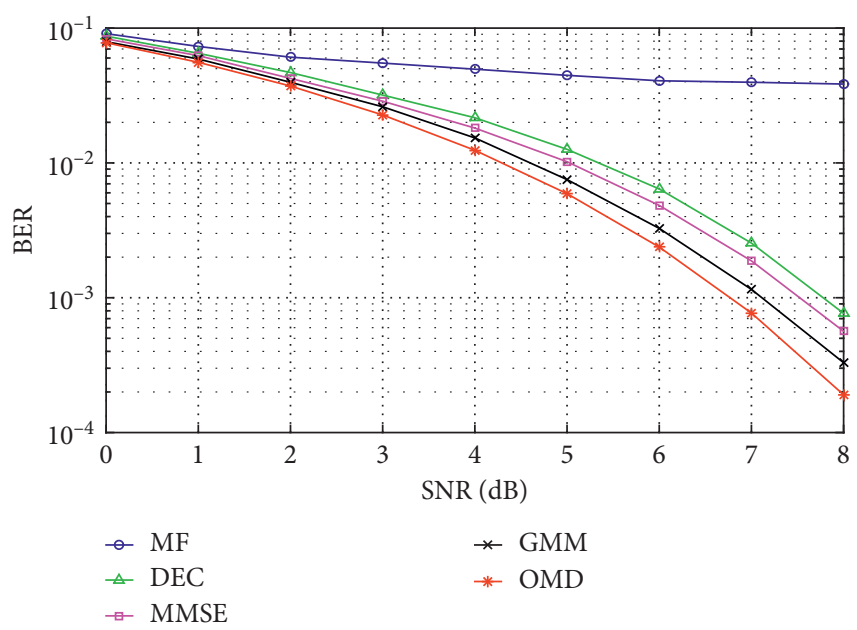

FIGURE 6: BER performance of various detectors with user number $K=30$. We compare Figure 6 (user number is 30 ) with Figure 5 (user number is 20) and Figure 4 (user number is 10).

capacity, which generally satisfies the spacecraft number of space formation flying applications.

5.3. Near-Far Resistance. Space formation flying system based on the UWB technology has limited power source without power control. The received power from interfering users may be much larger than the desired signals, which results in a near-far problem. The near-far resistance is a common performance measure for analyzing UWB systems. We evaluate the performance of GMM-MUD with respect to near-far resistance. We consider a 10-user DS-UWB system where near-far interference effect is present. Assume that the user 1 is the desired user and users $2-10$ are interfering users. The desired user's SNR is fixed on $6 \mathrm{~dB}$. The SNR of other users varies from $0 \mathrm{~dB}$ to $8 \mathrm{~dB}$ synchronously.

As shown in Figure 8, it is obvious that all the MUD detectors are unaffected by the near-far effect except the MF

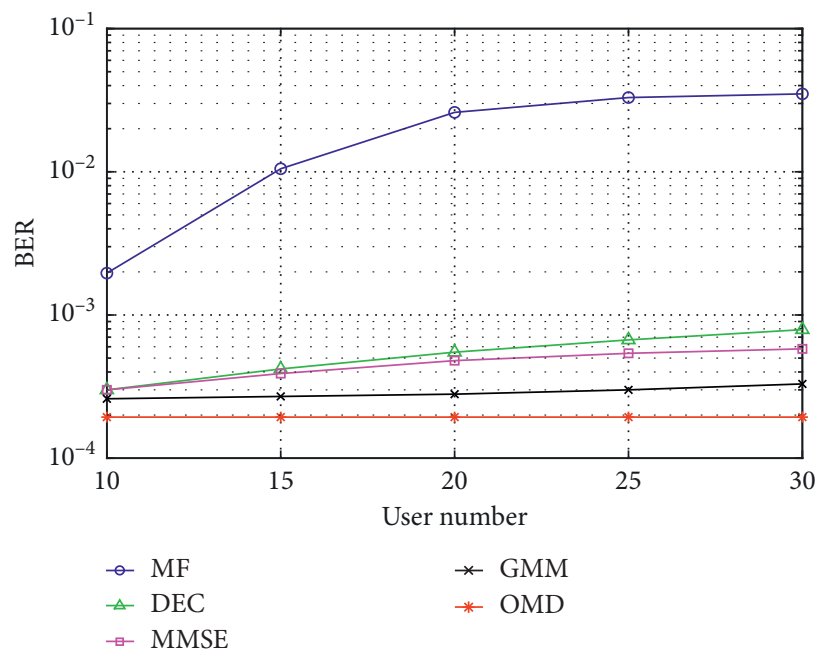

FIGURE 7: BER performance at $\mathrm{SNR}=8 \mathrm{~dB}$ for different number of users. The number of users increases from 10 to 30 and the value of SNR is $8 \mathrm{~dB}$.

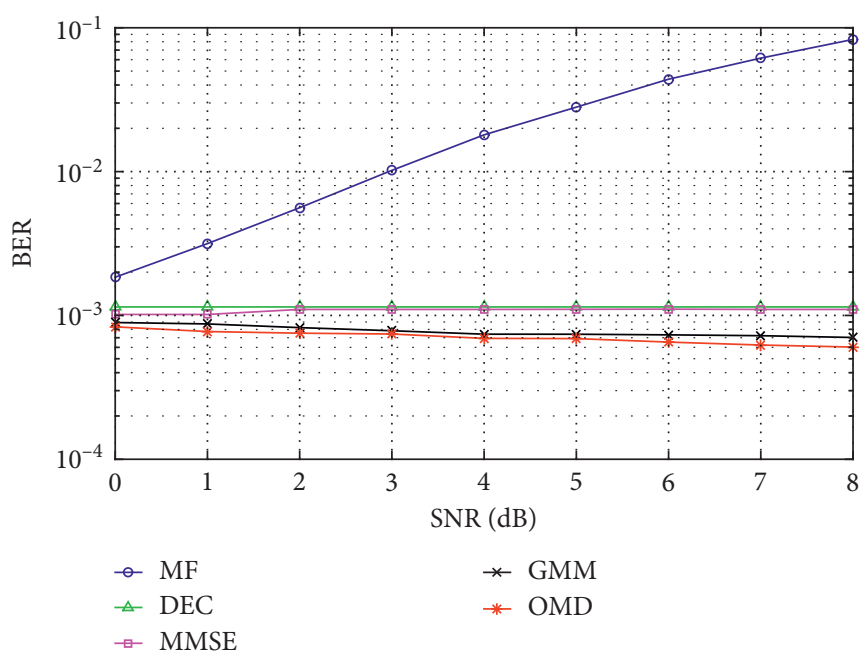

FIgURE 8: Near-far resistance with 10 users. We assume that the user 1 is the desired user $(\mathrm{SNR}=6 \mathrm{~dB})$ and users $2-10$ are interfering users (SNR varies from $0 \mathrm{~dB}$ to $8 \mathrm{~dB}$ ).

detector. Due to the good near-far resistance performance of the GMM-MUD detector approximating to that of the OMD detector, the proposed MUD algorithm is suitable for the DS-UWB-based space formation flying systems.

\section{Conclusions}

In this paper, an effective MUD algorithm called GMM-MUD for DS-UWB based space formation flying systems was proposed. The preprocessing based on mapping function was applied to the MUD detector in order to reveal the probability distributions of correct bits and error bits. The hypothesis testing problem used for judging error bits was described as GMM. EM algorithm was used to estimate the parameters of GMM. Error bits can be judged by GMM classification and revised by inverting the sign. Simulation results showed that 
the proposed MUD algorithm outperformed traditional suboptimal MUD algorithms. The user capacity of GMMMUD was large enough for space formation flying applications. Also, the proposed algorithm had good near-far resistance performance approximating to OMD. Moreover, the GMM-MUD detector had linear computational complexity with respect to the user number. Hence, the proposed GMMMUD algorithm was an effective MUD algorithm for DSUWB-based space formation flying systems.

\section{Data Availability}

The data used to support the findings of this study are available from the corresponding author upon request.

\section{Conflicts of Interest}

All the authors declare that there are no conflicts of interest regarding the publication of this paper.

\section{Acknowledgments}

This work was supported by the National Natural Science Foundation of China (grant numbers 61871157, 61471142, and 61102084).

\section{References}

[1] M. N. Sweeting, "Modern small satellites-changing the economics of space," Proceedings of the IEEE, vol. 106, no. 3, pp. 343-361, 2018.

[2] M. Xu and C. R. McInnes, "Wavelike patterns in precessing elliptical rings for swarming systems," Journal of Guidance, Control, and Dynamics, vol. 40, no. 7, pp. 1651-1663, 2017.

[3] M. Xu and C. R. McInnes, "Closed-loop control of the orbit evolution of "smart dust" swarms," Journal of Guidance, Control, and Dynamics, vol. 40, no. 7, pp. 1806-1814, 2017.

[4] Z. Dang and H. Zhang, "Linearized relative motion equations through orbital element differences for general Keplerian orbits," Astrodynamics, vol. 2, no. 3, pp. 201-215, 2018.

[5] R. Radhakrishnan, W. W. Edmonson, F. Afghah, R. M. Rodriguez-Osorio, F. Pinto, and S. C. Burleigh, "Survey of inter-satellite communication for small satellite systems: physical layer to network layer view," IEEE Communications Surveys and Tutorials, vol. 18, no. 4, pp. 2442-2473, 2016.

[6] E. Cianca, T. Rossi, A. Yahalom, Y. Pinhasi, J. Farserotu, and C. Sacchi, "EHF for satellite communications: the new broadband frontier," Proceedings of the IEEE, vol. 99, no. 11, pp. 1858-1881, 2011.

[7] M. D. Sanctis, E. Cianca, T. Rossi et al., "Waveform design solutions for EHF broadband satellite communications," IEEE Communications Magazine, vol. 53, no. 3, pp. 18-23, 2015.

[8] S. Niranjayan and N. C. Beaulieu, "Novel adaptive nonlinear receivers for UWB multiple access communications," IEEE Transactions on Wireless Communications, vol. 12, no. 5, pp. 2014-2023, 2013.

[9] H. Sasahara, K. Hayashi, and M. Nagahara, "Multiuser detection based on MAP estimation with sum-of-absolutevalues relaxation," IEEE Transactions on Signal Processing, vol. 65, no. 21, pp. 5621-5634, 2017.

[10] Q. Wang, G. Ren, and J. Wu, "A multiuser detection algorithm for random access procedure with the presence of carrier frequency offsets in LTE systems," IEEE Transactions on Communications, vol. 63, no. 9, pp. 3299-3312, 2015.

[11] X. Zhang, Y.-C. Liang, and J. Fang, "Novel bayesian inference algorithms for multiuser detection in M2M communications," IEEE Transactions on Vehicular Technology, vol. 66, no. 9, pp. 7833-7848, 2017.

[12] A. Barbieri, F. Pancaldi, and G. M. Vitetta, "A novel ultrawideband system for multiuser data communications," IEEE Transactions on Wireless Communications, vol. 10, no. 12, pp. 4324-4333, 2011.

[13] B. Shim, J. W. Choi, and I. Kang, "Towards the performance of ML and the complexity of MMSE: a hybrid approach for multiuser detection," IEEE Transactions on Wireless Communications, vol. 11, no. 7, pp. 2508-2519, 2012.

[14] E. Fishler and H. V. Poor, "Low-complexity multiuser detectors for time-hopping impulse-radio systems," IEEE Transactions on Signal Processing, vol. 52, no. 9, pp. 25612571, 2004.

[15] S. Poor and R. C. de Lamare, "Blind reduced-rank adaptive receivers for DS-UWB systems based on joint iterative optimization and the constrained constant modulus criterion," IEEE Transactions on Vehicular Technology, vol. 60, no. 6, pp. 2505-2518, 2011.

[16] R. C. de Lamare, M. Haardt, and R. Sampaio-Neto, "Blind adaptive constrained reduced-rank parameter estimation based on constant modulus design for CDMA interference suppression," IEEE Transactions on Signal Processing, vol. 56, no. 6, pp. 2470-2482, 2008.

[17] P. Liu and Z. Xu, "Blind MMSE-constrained multiuser detection," IEEE Transactions on Vehicular Technology, vol. 57, no. 1, pp. 608-615, 2008.

[18] G. Bartoli, N. C. Beaulieu, R. Fantacci, and D. Marabissi, "An effective multiuser detection scheme for MPR random access networks," IEEE Transactions on Communications, vol. 65, no. 3, pp. 1119-1130, 2017.

[19] P. S. Rossi and G. M. Kraidy, "Iterative multiuser detection for cooperative MIMO systems over quasi-static fading channels," IEEE Transactions on Wireless Communications, vol. 10, no. 11, pp. 3638-3643, 2011.

[20] M. G. Shayesteh, M. Menhaj, and H. Amindavar, "Computational intelligence techniques for multiuser detection of DS/ CDMA signals," in Proceedings of the IEEE Computational Intelligence in Robotics and Automation, vol. 1, pp. 390-395, Kobe, Japan, July 2003.

[21] K. P. Bagadi and S. Das, "Neural network-based adaptive multiuser detection schemes in SDMA-OFDM system for wireless application," Neural Computing and Applications, vol. 23, no. 3-4, pp. 1071-1082, 2013.

[22] K. Yen and L. Hanzo, "Genetic algorithm assisted joint multiuser symbol detection and fading channel estimation for synchronous CDMA systems," IEEE Journal on Selected Areas in Communications, vol. 19, no. 6, pp. 985-998, 2001.

[23] N. Zhao, Z. Wu, Y. Zhao, and T. Quan, "A population declining mutated ant colony optimization multiuser detector for MC-CDMA," IEEE Communications Letters, vol. 14, no. 6, pp. 497-499, 2010.

[24] Z. Yin, Z. Zong, H. Sun, Z. Wu, and Z. Yang, "A complexityperformance-balanced multiuser detector based on artificial fish swarm algorithm for DS-UWB systems in the AWGN and multipath environments," Journal on Advances in Signal Processing, vol. 2012, p. 229, 2012.

[25] H. Zhu and G. B. Giannakis, "Exploiting sparse user activity in multiuser detection," IEEE Transactions on Communications, vol. 59, no. 2, pp. 454-465, 2011. 
[26] B. Shim and B. Song, "Multiuser detection via compressive sensing," IEEE Communications Letters, vol. 16, no. 7, pp. 972-974, 2012.

[27] J. A. Tropp and A. C. Gilbert, "Signal recovery from random measurements via orthogonal matching pursuit," IEEE Transactions on Information Theory, vol. 53, no. 12, pp. 4655-4666, 2007.

[28] Y. Xie, Y. C. Eldar, and A. Goldsmith, "Reduced-dimension multiuser detection," IEEE Transactions on Information Theory, vol. 59, no. 6, pp. 3858-3874, 2013.

[29] Z. Yin, Y. Kuang, H. Sun, Z. Wu, and W. Tang, "A hybrid multiuser detection algorithm for outer space DS-UWB adhoc network with strong narrowband interference," KSII Transactions on Internet and Information Systems, vol. 6, no. 5, pp. 1316-1332, 2012. 


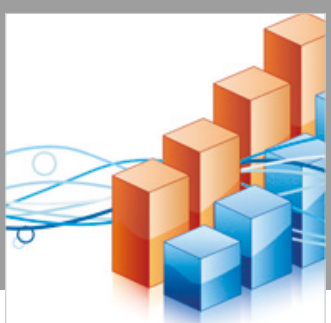

Advances in

Operations Research

\section{-n-m}
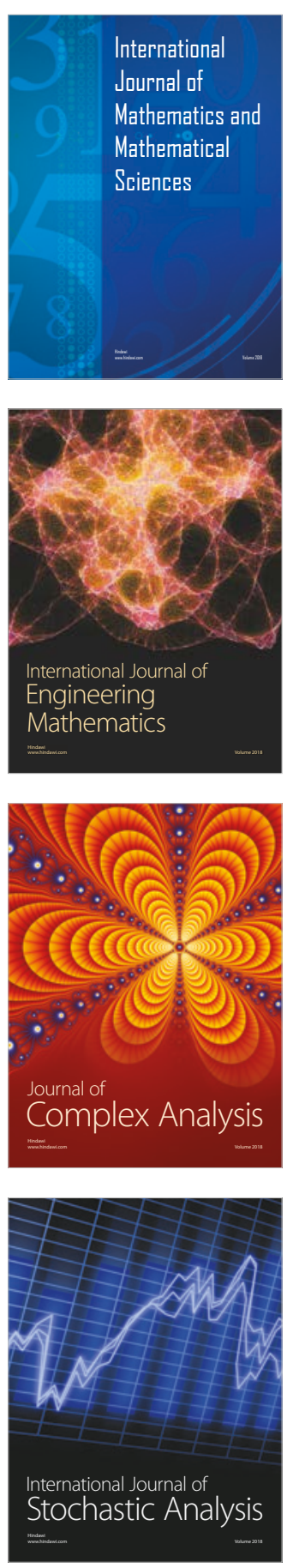
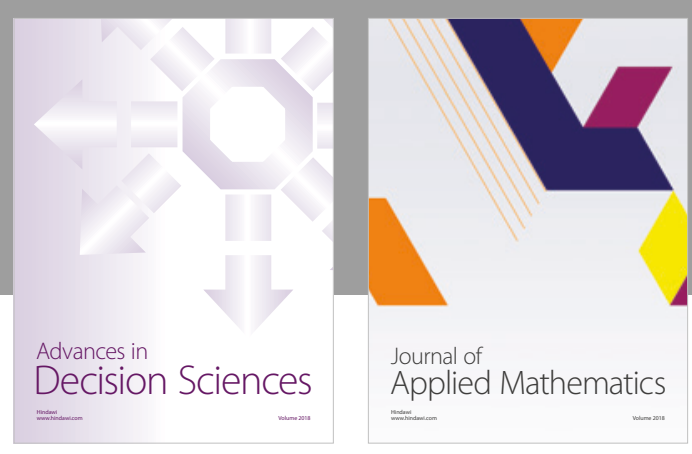

Journal of

Applied Mathematics
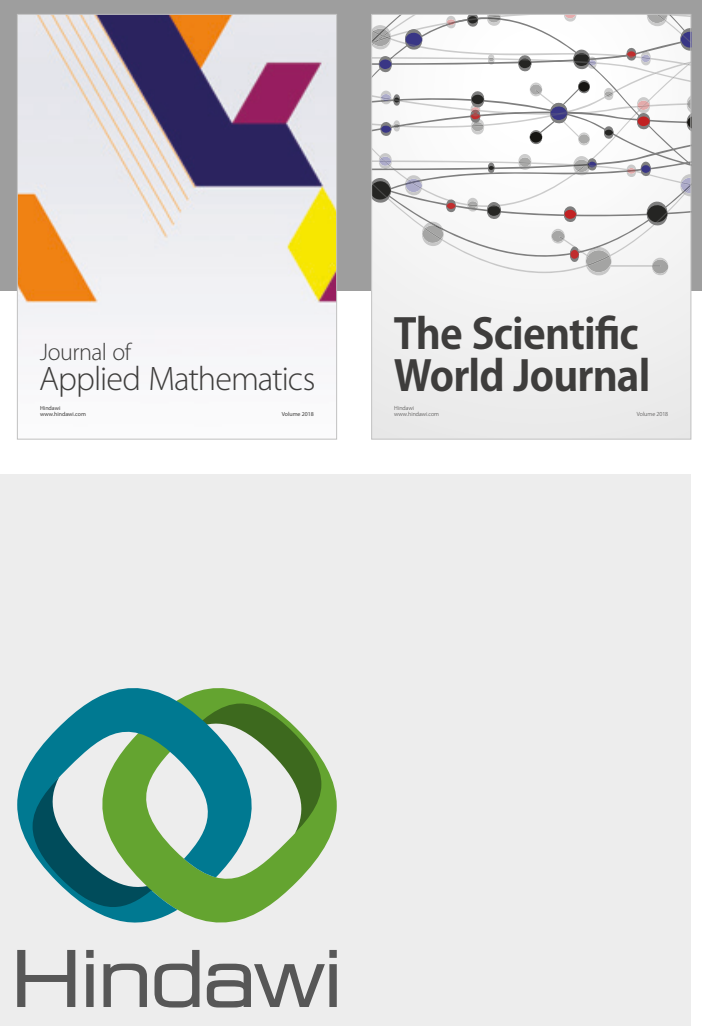

Submit your manuscripts at

www.hindawi.com

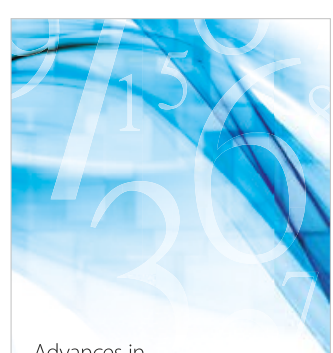

Advances in
Numerical Analysis
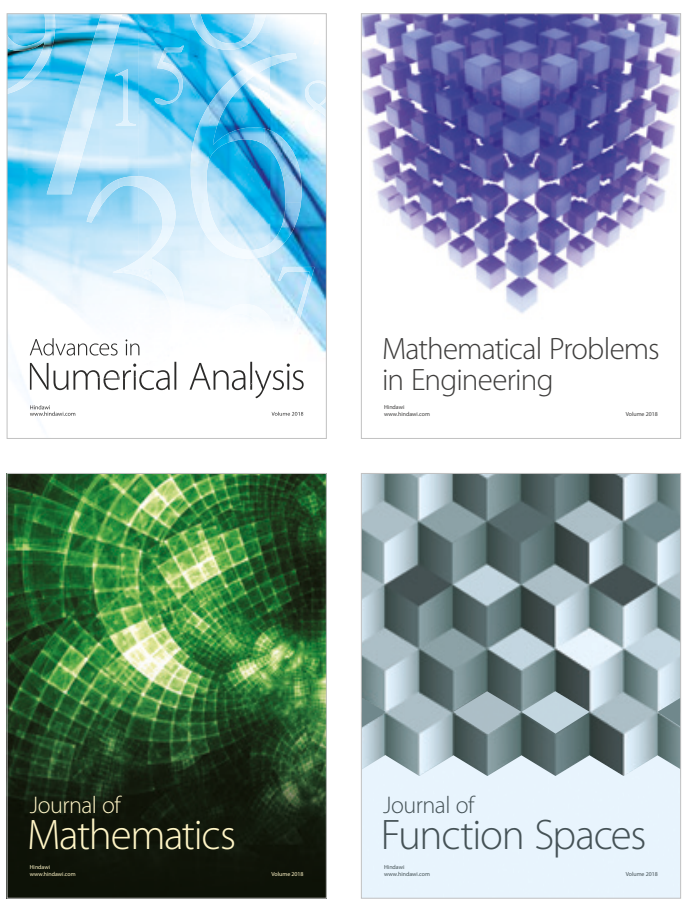

Mathematical Problems in Engineering

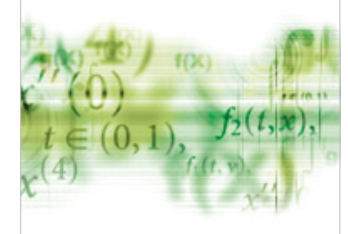

International Journal of

Differential Equations

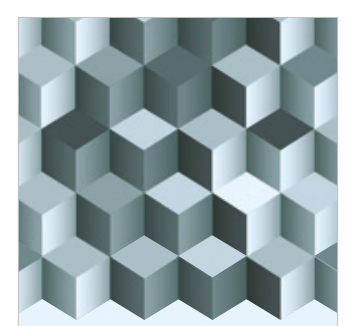

Journal of

Function Spaces

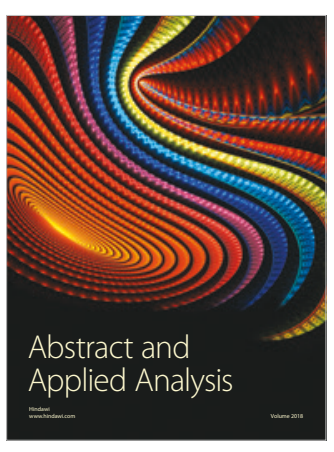

The Scientific

World Journal

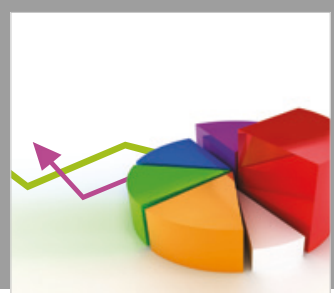

Journal of

Probability and Statistics
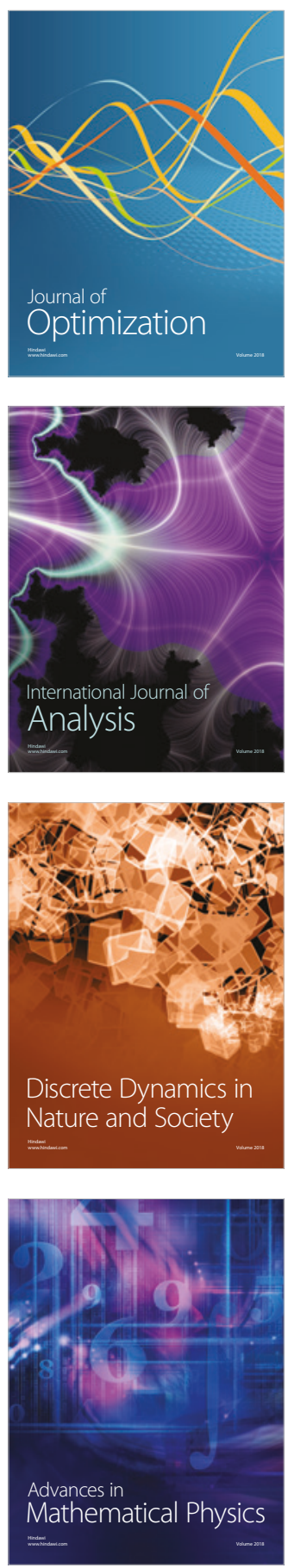